\title{
CHANGES OF THE ELECTROCARDIOGRAPHIC PARAMETERS DURING AGING IN CLINICALLY HEALTHY HOLSTEIN CATTLE
}

\author{
A. CHALMEH
}

School of Veterinary Medicine, Shiraz University, Shiraz, Iran

\begin{abstract}
Summary
Chalmeh, A., 2015. Changes of the electrocardiographic parameters during aging in clinically healthy Holstein cattle. Bulg. J. Vet. Med., 18, No 2, 105-111.

Electrocardiograms were recorded from 89 clinically healthy Holstein cattle divided into 10 age groups, comprising newborn calves (18 to 72 hours), 15 days, 1, 3, 6 and 9 months and 1, 3, 5, and 7 years of age. The electrocardiograms were recorded on a bipolar base apex lead, using limb lead I. Duration and amplitude of the P, QRS complexes, T waves and the PR, RR, QT and ST intervals were measured in traced electrocardiograms. The values of P-duration, P-R, R-R, Q-T and S-T intervals in adults were significantly higher than young cattle, but the amount of $\mathrm{P}$ and R-amplitudes in older animals were significantly lower than younger once $(\mathrm{P}<0.05)$. There were no significant changing patterns of T-duration, T, S and Q-amplitude among all age groups. With advancing age, degenerative changes such as developing fibrous tissue and fat deposits occured in heart muscle and its conduction system. Furthermore, the conductive properties of the body, cardiac mass and size, characteristics of Purkinje fibres in cardiac conductive system and changes of vagal tone during aging can explain the changing patterns of bovine electrocardiographic parameters at different ages. The present study, also, incorporated both calves and adult Holstein cows in a single wide study which can provide a good basis for judging the electrocardiograms in cattle.
\end{abstract}

Key words: aging, electrocardiographic parameters, Holstein cattle

\section{INTRODUCTION}

Electrocardiography as a noninvasive and inexpensive technique can be helpful to evaluate cardiac rhythm and conduction pathway performance in the heart musculature. Knowledge of the standard electrocardiographic parameters can be useful in order to evaluate the cardiac healthiness or sickness. Some cardiac abnormalities and arrhythmias are detectable by re- cording an electrocardiogram (ECG) and measuring the amplitudes, durations and intervals in the traced ECG (Radostits et al., 2007). Hence, information about these parameters in different ages of animals or human beings can assist clinicians in diagnosis and prognosis of the some cardiac problems. The potential use of electrocardiography in cattle is well recognised but 
there is no single comprehensive study on electrocardiographic parameters in different ages of this breed. Furthermore, several studies are performed on the effect of aging on electrocardiographic parameters in humans (Bachman et al., 1981; Jones et al., 1990; Macfarlane et al., 1994; Bansal \& Bansal, 2012), but no information exists regarding the evolution of the bovine ECG during aging. The present study was carried out in order to compare electrocardiographic parameters among different ages of clinically healthy Holstein cattle. We tried to express the changing patterns of the electrocardiographic parameters during aging in different ages of Holstein cattle as a bovine model.

\section{MATERIALS AND METHODS}

The present study was conducted from July 2009 till February 2010 on 89 clinically healthy Holstein cattle around Shiraz (latitude $29^{\circ} 33^{\prime} \mathrm{N}$ and longitude $052^{\circ} 36^{\prime} \mathrm{E}$, $1486 \mathrm{~m}$ above sea level), southwest Iran. The animals were assigned into 10 groups, comprising of newborn calves (18 to 72 hours), 15 days, 1, 3, 6 and 9 months and $1,3,5$, and 7 years old. The animals were examined prior to ECG recordings and were proved to be clinically healthy. None of the used animal in this study had any clinical signs of heart diseases (oedema, jugular distension or pulsation), and respiratory problems.

The ECGs were recorded on a bipolar base apex lead, using limb lead I. Animals were kept in a standing position in a stock without sedation and minimal restraint. No clipping or shaving was carried out for electrode attachment. When the animals were thought to be in a quiet state, the ECGs were recorded, using alligator-type electrodes which were attached to the skin after cleaning it with ethanol and applying electrocardiographic gel. The positive electrode (left arm) of lead I was attached to the skin of the left thorax at the fifth intercostal space, immediately caudal to the olecranon, and the negative electrode (right arm) was placed on the jugular furrow in the caudal third of the left neck (Radostits et al., 2007). The neutral electrode (right foot) was used on the skin of thoracic inlet. All ECGs were obtained on a single channel electrocardiographic machine (Kenz-line EKG 110, Suzuken Co., Ltd., Japan) with paper speed $25 \mathrm{~mm} / \mathrm{s}$ and calibration of $10 \mathrm{~mm}$ equal to $1 \mathrm{mV}$. Duration and amplitude of the P, QRS complexes and $\mathrm{T}$ waves and the PR, RR, QT and ST intervals were measured and averaged from successive beats following the usual conventions.

Mean and standard error of mean (SEM) were calculated for all parameters in different age groups. The comparison of each parameter in each group, with the same parameter in other age groups was analysed by the one way ANOVA with Tukey's post-hoc test, using SPSS software (SPSS for Windows, version 11.5, SPSS Inc, Chicago, Illinois). $\mathrm{P}<0.05$ was considered statistically significant.

\section{RESULTS}

The standard electrocardiographic parameters (mean \pm SEM) and the results of the one way ANOVA of each parameter among different age groups are shown in Table 1. The changing patterns of amplitudes, durations and intervals are presented in Fig. 1, 2 and 3, respectively. The results of the present study showed that the values of $\mathrm{P}$-duration, $\mathrm{P}-\mathrm{R}$ interval, $\mathrm{R}$ $\mathrm{R}$ interval, $\mathrm{Q}-\mathrm{T}$ interval and $\mathrm{S}-\mathrm{T}$ interval in adults were significantly higher than young cattle, but the $\mathrm{P}$ and $\mathrm{R}$ amplitudes 


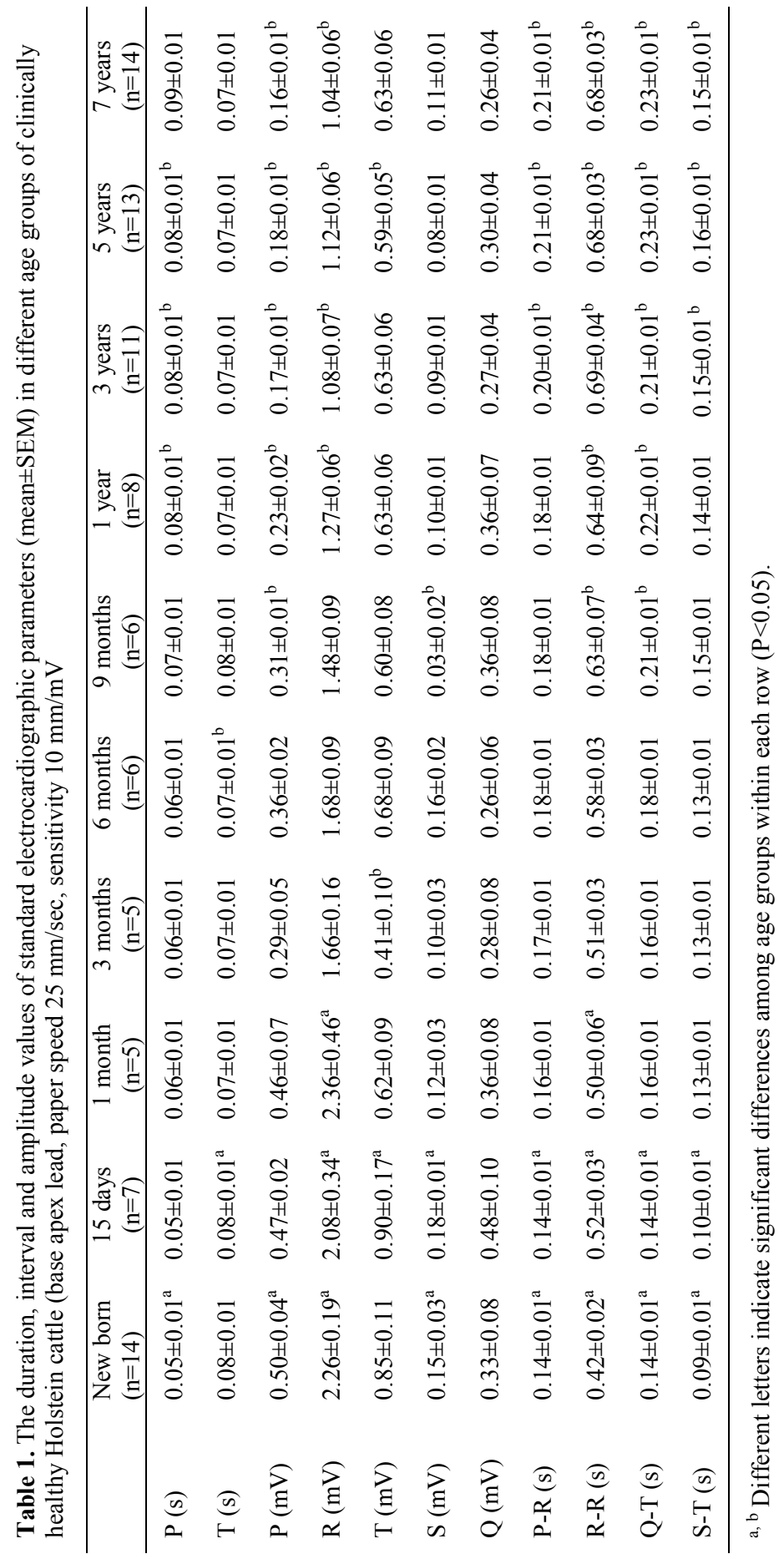

BJVM, 18, No 2 
in older animals was significantly lower than younger once $(\mathrm{P}<0.05)$.

There were no significant changing patterns of T-duration, T-amplitude, $\mathrm{S}$ amplitude and Q-amplitude among all age groups (Table 1).

Fig. 4 presents an ECG record of a clinically healthy one month-old calf.

\section{DISCUSSION}

The basic parameters of the standard bovine ECG which could be used as reference values are available in the consulted literature (Deroth, 1980; GhiŃă et al., 2008a, b; Pourjafar et al., 2012), but no comprehensive report was found on the changing patterns of electrocardiographic

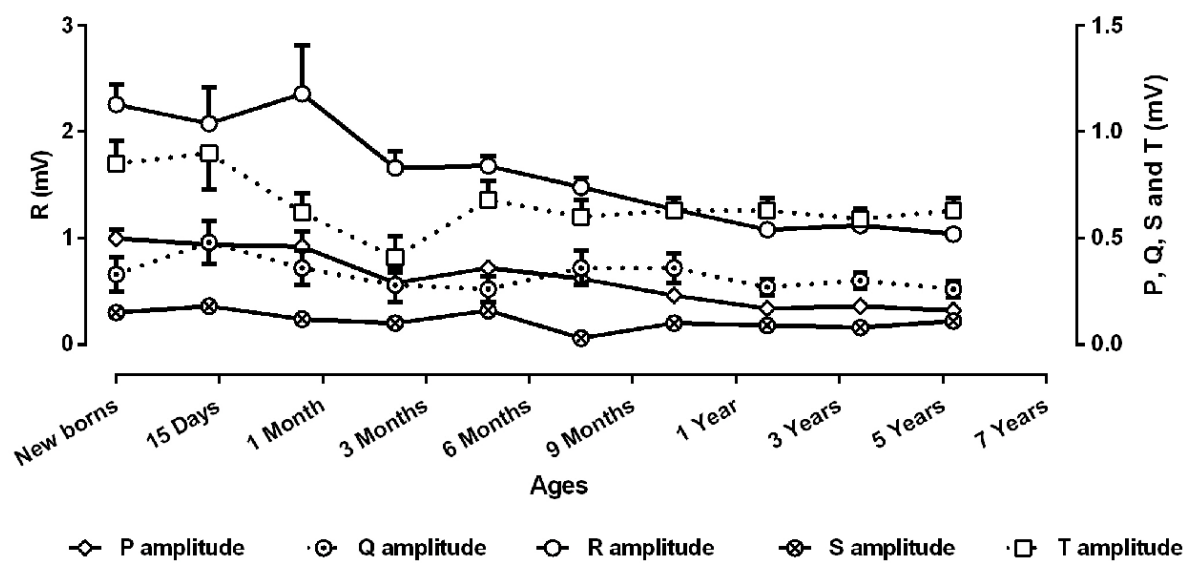

Fig. 1. Changing patterns of electrocardiographic waves amplitudes during aging of clinically healthy Holstein cattle (base apex lead, paper speed $25 \mathrm{~mm} / \mathrm{s}$, sensitivity $10 \mathrm{~mm} / \mathrm{mV}$ ).

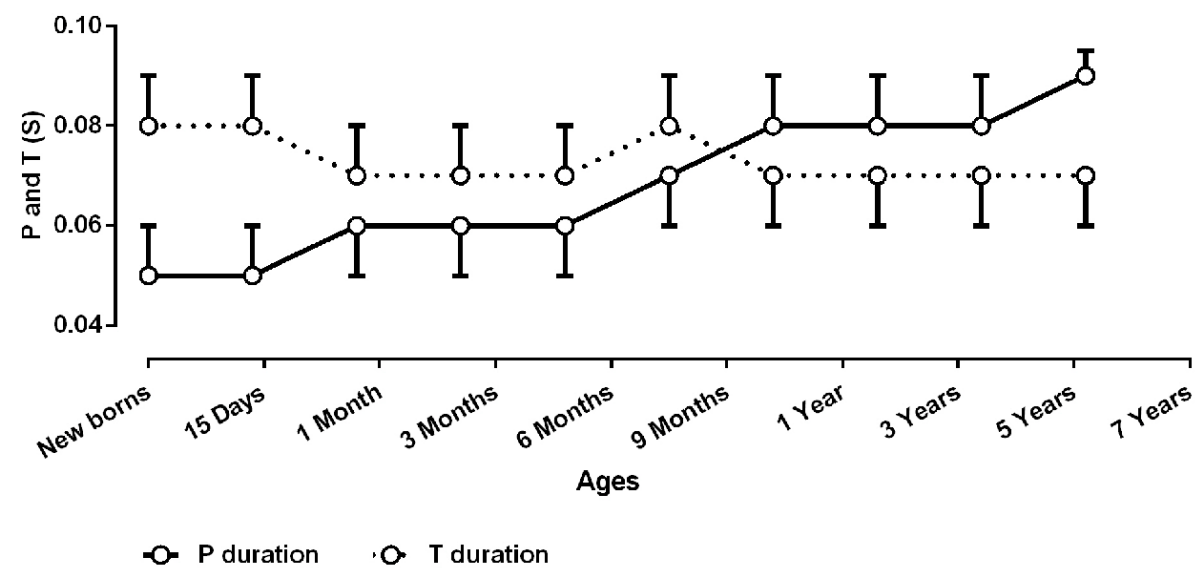

Fig. 2. Changing patterns of electrocardiographic waves durations during aging of clinically healthy Holstein cattle (base apex lead, paper speed $25 \mathrm{~mm} / \mathrm{s}$, sensitivity $10 \mathrm{~mm} / \mathrm{mV}$ ). 


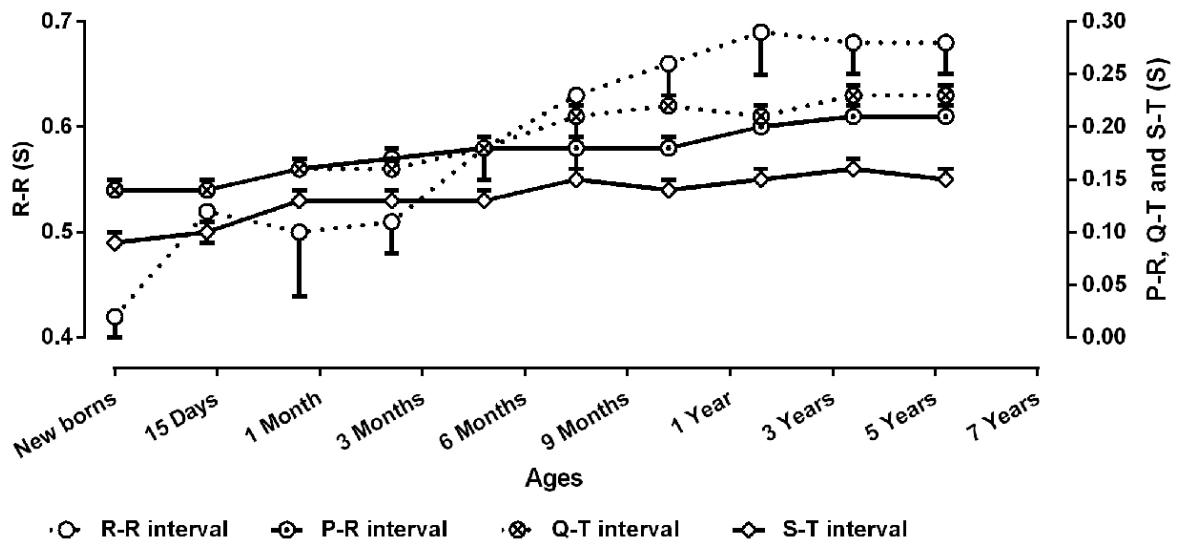

Fig. 3. Changing patterns of electrocardiographic waves intervals during aging of clinically healthy Holstein cattle (base apex lead, paper speed $25 \mathrm{~mm} / \mathrm{s}$, sensitivity $10 \mathrm{~mm} / \mathrm{mV}$ ).

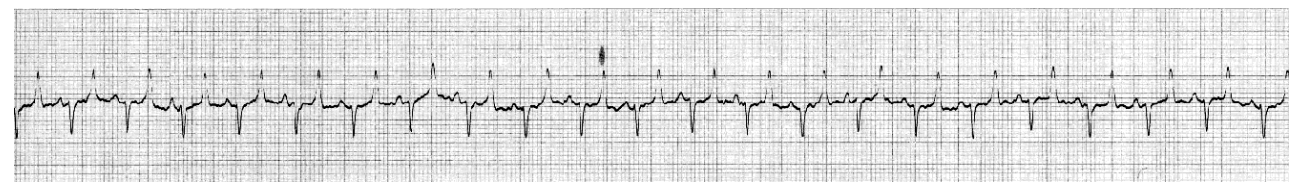

Fig. 4. ECG record from a clinically healthy 1-month-old Holstein calf (base apex lead system; paper speed $25 \mathrm{~mm} / \mathrm{s}$, sensitivity $10 \mathrm{~mm} / \mathrm{mV}$ ).

factors during aging in cattle. Furthermore, a comprehensive study on basic parameters of the standard bovine ECG, comprising all ages which could be used as reference values was not available in the consulted literature.

The results of the present study showed that in the studied Holstein cattle, some parameters changed during aging. Some parameters increased and some of them decreased. With advancing age, degenerative changes occur in heart muscle and its conduction system. Some of the pathways of pacemaker system may develop fibrous tissue and fat deposits (O'Connor et al., 2008). Furthermore, many of the changes that occur in the electrocardiogram reflect the anatomical dominance of the right ventricle during early months of life. At birth, the right ventricle is thick on account of high pulmonary artery pressure in utero. Falling pulmonary artery pressure after birth, causes decrease in the right ventricular pressure compared to the in utero period (Garson et al., 1998).

In the current study, amplitudes in younger animals were higher than in adults (Fig. 1). It may be suggested that the thinner chest wall in young animals allows the fast transmission of cardiac electrical waves to electrocardiographic equipment. The conductive properties of the body mass of ruminants, attributable to the volume of the gastrointestinal tract, also influence the distribution of body surface potentials comprising the ECG (Santamarina et al., 2001). It is possible 
that gradual development of body mass may cause difficulty in reaching the waves to the body surface due to relative electrical insulation by increasing body mass and changing in amplitudes can occur. It could be suggested that as the mass of heart in larger animals became larger in the process of growth, the duration of transfer of cardiac electrical activity also increases. Furthermore, ruminants have a deeply penetrating Purkinje system, and depolarisation from ventricular endocardium to epicardium occurs explosively and in many directions at once (Pennisi et al., 2002).

Our study indicated that the values of P-R, R-R, Q-T and S-T intervals were significantly higher in older animals than younger ones, which may probably be due to the larger size of the heart in older cows. With advancing age, widespread histologic changes in the conduction system occur. These changes may alter several features of the aging ECG, including duration of the PR and QT intervals, orientation of the electrical axis, duration and morphology of the atrial and ventricular complexes, and characteristics of the ventricular activity (Jones et al., 1990).

The R-R interval is the most obvious manifestation of age related variability within the neonatal ECG. This change can be accounted for by the gradual increase in vagal tone that accompanies aging. Younger animals also may be anxious during ECG acquisition, causing an artifactual decrease in the R-R interval (O'Connor et al., 2008).

Atrioventricular conduction is assessed via the P-R interval. Physiological prolongation of the P-R interval suggests conduction delay within the atrioventricular node. Large mass of cardiac musculature in adult cattle causes delay in heart conduction system (Radostits et al.,
2007). Studies on human ECG also represented that children have the P-R interval shorter than adults, presumably because of a lesser degree of cardiac muscle mass (Macfarlane et al., 1994).

The QRS duration in young animals is shorter than that of adults, once again, because of smaller cardiac muscle mass. In children, a mild prolongation of the QRS interval may occur without clinical correlate, which labelled as intraventricular conduction delay (O'Connor et al., 2008). The Q-T interval, measured from the beginning of the QRS complex to the termination of the $\mathrm{T}$ wave, represents ventricular activity. Macfarlane et al. (1994) reported that the Q-T interval increased during aging in human beings, which could be seen in aging cattle according to the present results.

Rezakhani et al. (2004) reported similar electrocardiographic values for healthy lactating cows and Deroth (1980) reported similar data but a slightly longer mean Q$\mathrm{T}$ interval in adult cattle. In the study of Pourjafar et al. (2012) the values of STsegment duration of the base apex lead in different ages are similar to our results.

The present study incorporated both calves and adult Holstein cows in different age groups to specifically clarify agerelated differences. These data can provide a better basis for judging the electrocardiograms in cattle. Our study on different normal electrocardiographic parameters may be helpful in standardising base apex lead in Holstein cattle of various ages in a comprehensive study.

\section{CONCLUSIONS}

According to our results, physiological changes of the electrocardiographic parameters occur during growing and aging of the Holstein calves. Furthermore, an 
understanding of normal developmental changes in the electrocardiogram in conjunction with clinical parameters will assist clinicians in better correlating electrocardiogram findings with the other patient clinical manifestations.

\section{ACKNOWLEDGMENT}

We would like to appreciate Mr. Davood Esfanyari for his valuable assistance in this study.

\section{REFERENCES}

Bachman, S., D. Sparrow \& L. K. Smith, 1981. Effect of aging on the electrocardiogram. American Journal of Cardiology, 48, 513-516.

Bansal, S. \& A. Bansal, 2012. Effect of age and sex on the R-R interval in ECG of healthy individuals. Indian Journal of Basic and Applied Medical Research, 1, 178-184.

Deroth, L., 1980. Electrocardiographic parameters in the normal lactating Holstein cow. Canadian Veterinary Journal, 21, 271-277.

Garson, Jr. A., J. T. Bricker \& D. J. Fisher, 1998. The Science and Practice of Pediatric Cardiology. Philadelphia: Lippincott Williams \& Wilkins, p. 89-101.

GhiŃă, M., G. Cotor \& C. Brăslasu, 2008a. The values of ECG parameters in newborn calves. Bulletin of the University of Agricultural Sciences and Veterinary Medicine Cluj-Napoca, 65, 473.

GhiŃă, M., G. Cotor, \& C. Brăslasu, 2008b. The values of some ECG components in dairy cows, using Dubois leads. Bulletin of the University of Agricultural Sciences and Veterinary Medicine Cluj-Napoca, 65, 474.

Jones, J., Z. M. Srodulski \& S. Romisher, 1990. The aging electrocardiogram. The American Journal of Emergency Medicine, 8, 240-245.

Macfarlane, P. W., S. C. McLaughlin, B. Devine \& T. F. Yang, 1994. Effects of age, sex, and race on ECG interval measurements. Journal of Electrocardiology, 27, 14-19.

O'Connor, M., N. McDaniel \& W. J. Brady, 2008. The pediatric electrocardiogram. Part I: Age-related interpretation. The American Journal of Emergency Medicine, 26, 221-228.

Pennisi, D. J., S. Rentschler, R.G. Gourdie, G.I. Fishman \& T. Mikawa, 2002. Induction and patterning of the cardiac conduction system. The International Journal of Developmental Biology, 46, 765-775.

Pourjafar, M., K. Badie \& A. Chalmeh, 2012. Evaluation of ST-segment duration and morphology of standard base apex lead electrocardiograms in different age groups of apparently healthy Holstein cattle. Journal of the Faculty of Veterinary Medicine, Istanbul University, 38, 51-57.

Radostits, O. M., C. C. Gay, K. W. Hinchcliff \& P. D. Constable, 2007. Diseases of the cardiovascular system. In: Veterinary Medicine: A Textbook of the Diseases of Cattle, Horses, Sheep, Pigs and Goats. $10^{\text {th }}$ edn, Elsevier, pp. 399-438.

Rezakhani A., A. A. Papahn \& S. Shekarforoush, 2004. Analysis of base apex lead electrocardiograms of normal dairy cows. Veterinarski Arhiv, 74, 351-358.

Santamarina, G., L. Espino \& L. M. Suarez, 2001. Electrocardiographic parameters of free-ranging roe deer (Capreolus capreolus). Journal of Zoo and WildLife Medicine, 32, 441-446.

Paper received 18.11.2013; accepted for publication 25.04.2014

\section{Correspondence:}

Chalmeh Aliasghar Department of Clinical Sciences, School of Veterinary Medicine, Shiraz University, Shiraz, Iran, P. O. Box: 71345; tel: +98 7116138700 ; fax: +98 7112286940 ; e-mail: achalmeh81@gmail.com 